\title{
A compact and accurate set of basis functions for model-based reconstructions
}

Christina Eckel, Otto-von-Guericke University Magdeburg, Magdeburg, Germany, christina.eckel@st.ovgu.de Sebastian Bannasch, Institute for Medical Engineering, Otto-von-Guericke University Magdeburg, Magdeburg, Germany, sebastian.bannasch@ovgu.de

Robert Frysch, Institute for Medical Engineering, Otto-von-Guericke University Magdeburg, Magdeburg, Germany, robert.frysch@ovgu.de

Georg Rose, Institute for Medical Engineering, Otto-von-Guericke University Magdeburg, Magdeburg, Germany, georg.rose@forschungscampus-stimulate.de

Purpose: Model-based perfusion reconstruction (MBPR) by using a weighting sum of basis functions, is used to describe the dynamic contrast agent distribution by superposition of incorporated prior knowledge. It handles temporal under-sampling in measurements acquired with a slowly rotating X-ray-based imaging system, in our case a C-arm based computed tomography (CT). However, here challenging issue arises that the computing complexity increases proportional to the number of prior knowledge elements. Thus, the aim of this study is to analyze clinical data and elaborate basis functions, that maps various patients with a small orthonormal basis set (ONB).

Materials \& Methods: This work is based on five reconstructed clinical perfusion CT data sets. For each patient, regions of interest were manually figured out in order to enhance the content. Therefore, bones and catheters have to be removed out of the data, to prevent a falsification of the curves by them. The principal component analysis (PCA) to compress the relevant information of perfusion and create an ONB was used. In order to achieve an ONB which also optimal maps unknown patients, the cross validation method was used, i.e. the datasets of four patients were utilized for the estimation of the ONB, while the remaining patient was used for evaluation. The target ONB is successively optimized by eliminating redundant dynamics. Finally, the ONB gets evaluated by the mean-absolute-percentage-error (MAPE) of MBPR.

Results: A compact ONB with three basis functions that maps all five patients without a significant deviation of the approximated curves and the original ones is obtained. Especially, regions with high blood supply can be reconstructed very accurately and a reduction of noise is qualitatively visible in the image.

Conclusion: An optimum ONB for the MBPR requires that the curves can be modeled as exactly as possible with a few basis elements. The use of only a few elements also leads to short computing times. In this work a good approximation of the curves with three basis elements is received. This results in an improved MBPR that in turn can lead to a higher precision of stroke diagnostics and treatments by using C-Arm CT. To take statistic fluctuations into account and obtain more robust ONBs, a greater amount of data sets will be used in further work.

Acknowledgements: The work of this paper is funded by the Federal Ministry of Education and Research within the Forschungscampus STIMULATE under grant number '13GW0095A'. Additionally, the authors thank Professor Dr. Thomas Henzler and the $\mathrm{M}^{2} \mathrm{OLIE}$ Forschungscampus for providing the CT data sets. 


\section{Volumetric 3D stitching of optical coherence tomography volumes}

Max-Heinrich Laves, Leibniz Universität Hannover, Institute of Mechatronic Systems, Hannover, Germany, laves@imes.uni-hannover.de

Lüder A. Kahrs, Leibniz Universität Hannover, Institute of Mechatronic Systems, Hannover, Germany,

kahrs@imes.uni-hannover.de

Tobias Ortmaier, Leibniz Universität Hannover, Institute of Mechatronic Systems, Hannover, Germany, ortmaier@imes.uni-hannover.de

Optical coherence tomography (OCT) is a non-invasive medical imaging modality, which provides high-resolution transectional images of biological tissue. However, its potential is limited due to a relatively small field of view. To overcome this drawback, we describe a scheme for fully automated stitching of multiple 3D OCT volumes for panoramic imaging.

The voxel displacements between two adjacent images are calculated by extending the Lucas-Kanade optical flow algorithm to dense volumetric images. A RANSAC robust estimator is used to obtain rigid transformations out of the resulting flow vectors. The images are transformed into the same coordinate frame and overlapping areas are blended.

The accuracy of the proposed stitching scheme is evaluated on two datasets of 7 and 4 OCT volumes, respectively. By placing the specimens on a high-accuracy motorized translational stage, ground truth transformations are available. This results in a mean translational error between two adjacent volumes of $16.6+/-0.8$ micrometers $(2.8+/-0.13$ voxels $)$.

To the author's knowledge, this is the first reported stitching of multiple 3D OCT volumes by using dense voxel information in the registration process. The achieved results are sufficient for providing high accuracy OCT panoramic images. Combined with a recently available high-speed 4D OCT, our method enables interactive stitching of hand-guided acquisitions. 


\section{Characterization and validation of camera system and illumination during In- traoperative Hyperspectral Imaging in neurosurgery}

Richard Mühle, Klinik und Poliklinik für Neurochirurgie, Universitätsklinikum Dresden, Dresden, Deutschland, richard.muehle@tu-dresden.de

Stephan B. Sobottka, Klinik und Poliklinik für Neurochirurgie, Universitätsklinikum Dresden, Dresden, Deutschland, stephan.sobottka@uniklinikum-dresden.de

Ute Morgenstern, Institut für Biomedizinische Technik, Technische Universität Dresden, Dresden, Deutschland, ute.morgenstern@tu-dresden.de

To successfully perform a resection of brain tumor the physician need preoperative examination based on MRI, intraoperative information on tumor borders, and brain tissue functionality. To identify functional areas of increased metabolism, Intraoperative Optical Imaging (IOI) technology can be used to generate two-dimensional activity maps. IOI uses a specialized hardware and software setup that analyses selected wavelengths or broadband spectra (RGB) of the reflected light from the white-light exposed cortex. In contrast, hyperspectral imaging (HSI) expands the procedure to the entire continuous light spectrum in order to obtain further information on brain tissue types and properties. An HSI system with an intraoperatively usable HSI hardware setup, based on a standard surgical microscope, a halogen light source, and a pushbroom HSI camera system which records quasi-continuous light spectrum wavelengths between 500 and $1000 \mathrm{~nm}$ with a resolution of $5 \mathrm{~nm}$, has been developed and subcomponents are examined. The HSI system was attached to the surgical microscope using a standard beam splitter, and the illumination was coupled via fiber-optic system. Via beam splitter an RGB camera was applied to adjust focus and zoom. Problems of characterization and validation of the HSI camera and the illumination are discussed. 40 data cubes for pixel- and wavelength-specific SNR calculation under neurosurgical intervention conditions are processed to assess reproducibility of image data acquisition. In addition, the warming of the surface of non-perfused and perfused porcine kidneys as well as of non-perfused porcine brain are examined. A first validation of the intraoperative hyperspectral imaging hardware setup shows that the intraoperatively recorded data can be evaluated without any fundamental limitations. Hyperspectral imaging can be easily integrated into the workflow of an operation without any noticeable change in clinical setup. 


\section{Enhancement of Region of Interest CT Reconstructions through Multimodal Data}

*David Schote, Tim Pfeiffer, Georg Rose, Otto von Guericke University, Germany

Computed tomography (CT) scans are frequently used intraoperatively, for example to control the positioning of implants during intervention. Oftentimes, a full field of view is often unnecessary to provide the required information. Instead, so-called region-of-interest (ROI) imaging can be performed, which allows for substantial reduction in the applied X-ray dose. However, ROI imaging leads to data inconsistencies, caused by the truncation of the projections. This lack of information severely impairs the quality of the reconstructed images.

This study presents a proof-of-concept for a new approach that combines the incomplete CT data with ultrasound (US) data and time of flight (ToF) measurements in order to restore some of the lacking information. In a first step, the object shape is extracted from the ToF scane and the resulting silhouette is filled with an initial estimate for the absorption coefficient. Using a forward projection, the sinogram of this object is computed and used to extrapolate the missing parts in the original ROI projections. In the second step, the US information is processed. To do so, the US image is registered to the reconstruction from the projections that have been extrapolated using ToF data in the first step. The registered US image is segmented and segments are filled with estimates for their individual absorption coefficients (e.g. bone or soft tissue). Finally, a refined extrapolation is computed based on the forward projection of the processed US image.

The routine is evaluated in a simulation study using the original Shepp-Logan phantom in ROI cases with different degree of truncation. Image quality is assessed by means of normalized root mean square error. The proposed method significantly reduces truncation artifacts in the reconstructions and achieves considerable radiation exposure reductions. Future work will address further optimizations in terms of image registration and the application of the proposed method to real data. 


\section{Intervention assessment tool for primary tumors in the liver}

Cristina Oyarzun Laura, Visual Healthcare Technologies, Fraunhofer IGD, Darmstadt, Germany, cristina.oyarzun@igd.fraunhofer.de

Klaus Drechsler, Aachen University of Applied Sciences, Aachen, Germany, drechsler@fh-aachen.de

Marius Erdt, Fraunhofer IDM@NTU, Singapore, Singapore, marius.erdt@ fraunhofer.sg

Stefan Wesarg, Visual Healthcare Technologies, Fraunhofer IGD, Darmstadt, Germany, stefan.wesarg@igd.fraunhofer.de

Reto Bale, University Clinic of Radiology, Innsbruck Medical University, Innsbruck, Austria, reto.bale@i-med.ac.at

After a liver tumor intervention the medical doctor has to compare both pre and postoperative CT acquisition to ensure that all carcinogenic cells are destroyed. A correct assessment of the intervention is of vital importance, since it will reduce the probability of tumor recurrence. In the clinical practice the only help for medical doctors to ensure the success of the intervention is a side by side visualization of both images. However, the liver is composed of soft tissue and will be subject to high deformations due to breathing and intervention. This further complicates the assessment process. In this sense some methods have been proposed to support medical doctors. All of them are focused on secondary tumors which are visible in the portal phase. Unfortunately, there exist tumors, namely, primary tumors and in concrete hepatocellular carcinomas that are often only visible in the arterial phase. We propose an intervention assessment tool that can handle both types of tumors. First, a Demons based multiphase registration is carried out to register both preoperative CT acquisitions (arterial and portal phases). This way all tumors appear registered to the preoperative portal phase CT. Then, the liver and vascular trees of preoperative and postoperative CTs are segmented and a landmark based registration is carried out. After this last step both primary and secondary tumors are registered to the postoperative CT. The contour of the registered tumor is visualized over the postoperative CT. This way the medical doctor can directly compare the tumor and the area of the intervention. The areas that were not covered by the intervention or that did not keep the required safety margin are visualized in red and yellow respectively. The rest is visualized in green. The method has been tested in 9 patients, with an average registration accuracy of $1.41 \mathrm{~mm}$. 


\section{Motility analysis by means of video tracked markers}

Kornelius Lente, inomed Medizintechnik GmbH, Emmendingen, Germany, K.Lente@inomed.com

Karin H. Somerlik-Fuchs, inomed Medizintechnik GmbH, Emmendingen, Germany, K.Somerlik@inomed.com

Jonas F. Schiemer, University Medicine of the Johannes Gutenberg-University Mainz, Mainz, Germany, jonasfriedrich.schiemer@unimedizin-mainz.de

Axel Heimann, University Medicine of the Johannes Gutenberg-University Mainz, Mainz, Germany, Axel.Heimann@unimedizin-mainz.de

Roman Ruff, Fraunhofer IBMT, Sulzbach, Germany, Roman.Ruff@ibmt.fraunhofer.de

Jan Baumgart, University Medicine of the Johannes Gutenberg-University Mainz, Mainz, Germany, jan.baumgart@unimedizin-mainz.de

Klaus-Peter Hoffmann, Fraunhofer IBMT, Sulzbach, Germany, Klaus-Peter.Hoffmann@ibmt.fraunhofer.de

Thilo B. Krüger, inomed Medizintechnik GmbH, Emmendingen, Germany, T.Krueger@inomed.com

Werner Kneist, University Medicine of the Johannes Gutenberg-University Mainz, Mainz, Germany, werner.kneist@ unimedizin-mainz.de

The motility of the gastrointestinal tract is crucial for digestive activity and dysfunction can lead to severe disease pattern. A method for analysing the motility is needed when treatment approaches shall be evaluated. Therefore markers attached to different locations on the stomach and the bowel of pigs are video tracked in this research study. The markers are designed to provide a high contrast and have an adhesive side for fixation. Above the operation field a video camera has been placed to film the markers during the procedure. To analyse the video data a special algorithm has been implemented. The algorithm requires a registration process at the beginning of each recording which allows the parallel tracking of multiple markers. After the registration the algorithm tracks the position of the marker frame by frame. Each frame is converted into a greyscale picture by adding specified colour values of each pixel. This allows emphasizing certain colours. The centre of the marker is determined by computing the horizontal and vertical centre of the marker starting at the corresponding marker position of the previous frame. After completion the data is stored as coordinates and a video with the marker position displayed for further processing. For advanced analysis the data can be synchronized with electromyography signals, for example. The marked videos show a promising tracking of the markers. However, if the algorithm loses track of a marker during a recording, it is unlikely to relocate it due to the successive processing of the frames. Nevertheless this method provides a simple and easy to use solution for movement detection of the gastrointestinal tract. 


\section{Bi - Domain Intraoperative Registration of Vessels}

Ady Naber, Karlsruhe Institute of Technology (KIT), Institute of Biomedical Engineering (IBT), Karlsruhe, Germany, ady.naber@kit.edu

Werner Nahm, Karlsruhe Institute of Technology (KIT), Institute of Biomedical Engineering (IBT), Karlsruhe, Germany, werner.nahm@kit.edu

The segmentation and registration of structures are gaining importance due to the increasing demand of auto- mated image enhancement and understanding. Especially in medicine and life science, assistance systems could have a large impact on diagnosis, treatment and quality control. Dye driven procedures, such as uorescence imaging with Indocya- nine green (ICG), are nowadays indispensable because they enhance contrast, reveal structures and deliver the operator with important information. The contact free ICG angiography is providing the surgeon spatial and temporal information on blood ow within a vessel. The processing of those informa- tion is done manually or semi automated but is very helpful for the surgeon. Extending the degree of automatism, the amount of information processed and even augment or transfer it into another domain could deliver the operator useful support and improve surgical work ow. Using, analyzing and transferring those information from ICG-IR domain into the RGB domain is the focus of this project. We are introducing a vessel regis- tration method in the RGB domain driven by the spatial $u$ - orescence behavior of the vessel in the ICG-IR domain. The method includes Superpixel based segmentation of the vessel in the ICG-IR domain, the spatial gradient based transfer and registration in the RGB domain and the continuous segmen- tation of the vessel in a RGB video. This paper show a proof of concept of the method. The results show an successful in- ter domain information transfer and registration of the vessel. Further tracking of the vessel over all frames is possible. Nev- ertheless limitations are revealed and discussed. 